Gazi University
Journal of Science
$\mathrm{http} / /$ dergipark.gov.tr/gujs

\title{
Sustainable Management of a Renewable Fishery Resource with Depensation Dynamics from a Control Systems Perspective
}

\author{
Oguzhan CIFDALOZ* \\ Cankaya University, Electrical - Electronics Engineering Department, 06790, Ankara, Turkey
}

\author{
Highlights \\ - Control systems provides robust and implementable solutions to management of renewable resources. \\ - Optimal solution to management problem is difficult to implement and vulnerable to uncertainties. \\ -Well-established tools and methods of control systems discipline proposes alternative solutions.
}

\section{Article Info}

Received: 15 Mar 2021

Accepted: 17 Aug 2021

\section{Keywords}

Sustainability

Social-ecological

systems

Optimal control

Renewable resource

management

\begin{abstract}
Human societies are exploiting natural renewable sources such as fisheries, forests, groundwater basins, rivers, and soil at an increasing intensity. Around the world, these resources are being managed by various institutions or governments. One of the challenges faced by institutions is to develop strategies and policies to effectively manage these renewable resources under social and ecological uncertainties, disturbances, policy implementation difficulties, and measurement errors. In this paper, a fishery is considered as an example and the problem of managing a fishery is approached from a control systems perspective. The justification behind this approach is due to the observation that the problem of managing a renewable resource can be posed as a control systems problem and that the discipline of control systems possesses tools and methods to deal with model uncertainties, external disturbances, measurement errors and implementation issues. For the fishery, a depensation type population dynamics model is considered. Depensatory models are used in social/ecological systems in order to model dynamics of certain species of fish populations. An optimal control strategy based on Pontryagin's Maximum Principle is derived and its sustainability and robustness properties with respect to parametric uncertainties, measurement errors and disturbances are examined. Finally, a sub-optimal but more robust control strategy is proposed and its robustness properties are provided. The main objective of the paper is to show that a control systems engineering approach can be applied to a social-ecological problem and it can provide easy to implement management strategies, insight, and guidance into the management of renewable resources.
\end{abstract}

\section{INTRODUCTION}

Sustainability is concerned with satisfying the demands of human beings without endangering future generations. Amid increasing world population, diminishing agricultural lands, dwindling fresh water resources, and rapid marine pollution, sustainability is becoming more and more crucial for the well-being of societies. It has three major components: Economic, environmental, and social. In this paper, sustainability of renewable resources, specifically a fishery, is investigated from the perspective of control systems. The objective of this paper is to argue that the well-established procedures of control engineering discipline can be applied to managing and/or providing guidance to managing renewable resources.

Renewable resource management problems are studied within the scope of social-ecological systems. Social dynamics include the behaviour of agents (people, organizations) and the economic incentives of these agents. Ecological dynamics include the habitat that the resource inhabits. Hence, these systems are intimately coupled with the environmental issues associated with resource exploitation. As a consequence, studying social-ecological systems involve population dynamics of the resource, its interactions with the 
environment that it inhabits, but also the economic dynamics of harvesting the resource, and the economic attitudes of the resource exploiters. Despite efforts by governments and/or institutions renewable resources are managed poorly around the globe including fisheries [1-5], forests [6-8], groundwater basins [9-11], and soil $[12,13]$. Various explanations for the cause of poor management are suggested, but they can be grouped into two broad categories: $i$ ) ignoring the actual economic motives of economic agents and $i$ ) uncertainty associated with the dynamics of the renewable resources in consideration [1]. This demonstrates that institutions need to devise policies capable of effectively managing renewable resources subject to high levels of uncertainty.

A frequent approach to such management problems is stochastic optimization. Studies which take into account a single specific source of uncertainty include the size of the resource stock (population) [14-20], the number of new recruits or price [21-23]. However, since stochastic optimization requires to assign probabilities to possible outcomes, it can be restrictive. Such models generate knowledge and understanding about the uncertainties associated with renewable resources, but their contribution to actually managing the resource is somewhat limited.

In order to manage a real-world resource, at least three actions need to be taken: objectives should be determined, practical implementation issues should be resolved, and a continuous modeling (identification) should be undertaken. Some researchers believe that practical implementation issues are at the root of the failures of resource (e.g. fisheries) management [1, 2, 24].

In this paper, a fishery management problem is approached from a robust control systems perspective. Publications which emphasize the importance of modeling, feedback, and analysis have started to appear in literature in the last decade, for instance in modeling renewable resource dynamics $[25,26]$, control of renewable resources [27-31], and analysis of management implementations [32-34].

The main premise of this paper is to advocate that well-established control systems design tools and procedures can provide robust and easily implementable solutions to the management of fisheries. Before the negative impact of over exploitation on sustainability of fisheries was realized and recognized as a serious problem, fisheries were unregulated open-access fisheries, in which fishermen make individual assessments about the profitability of the resource and decide whether to harvest the resource or not at their own discretion. Although there still exist open-access fisheries around the world, most fisheries are actively regulated by institutions and governments today via seasonal fishing bans, landing quotas, or technology restrictions. As described in the literature survey, once the importance of feedback has been acknowledged by the managing institutions, the focus of analysis has largely been based on optimal management ideas. Publications in the sustainability literature [27-34] state the problems associated with the optimal control strategy, although, not written from a systems perspective and without discussing alternative management strategies.

On the other hand, the discipline of control systems is already equipped with tools and methods to quantify the robustness and vulnerability of social ecological systems (such as the fishery management problem) via powerful time and frequency domain analyses, and provides a myriad of alternative controller design strategies. To this purpose, analysis of a benchmark case, namely the depensation model of a fishery is provided and analyzed. Natural dynamics of a resource without harvesting and when subject to harvesting is investigated via equilibrium analysis. Based on the model and a specified cost function, an optimal control (management) strategy is developed and the derivation of the optimal control is described. Robustness properties and vulnerabilities of this optimal control strategy in the context of managing fisheries for the depensation model are demonstrated via its sensitivity to various parameter uncertainties, measurement errors and disturbances. Next, a dynamic sub-optimal control (management) strategy is proposed and the strengths of the sub-optimal control strategy is analyzed subject to the same parameter uncertainties, measurement errors and disturbances. It is shown that even simple control structures provide better performance with respect to the aforementioned sources of error. It is concluded that control engineering perspective can be very beneficial in the management of renewable resources. 
In what follows, Section 2 describes the problem to be addressed and defines the resource dynamical model. Section 3 defines the optimal and sub-optimal control structures. Section 4 presents a numerical example to further explain and convey the concepts and ideas. Section 5 discusses the results and presents directions for future research.

\section{PROBLEM STATEMENT AND SYSTEM MODEL}

The problem addressed in this paper is the managing or controlling a renewable fish resource where contradicting objectives need to be satisfied. Managing any renewable resource has trade-offs, very much like the trade-offs that are encountered in control systems problems solved in electrical engineering. Consider the case where the resource is a fishery. The resource has some economic value. The resource exploiters, i.e. the fishermen or fishing companies, would like catch fish and sell it in the market to obtain economic gain. However, when not managed or poorly managed, fishing activity quickly depletes the resource and drives the fish population to extinction, which results in unsustainable economic gain and a dire cost to ecology. A management strategy, or in engineering terms, a control system is required to maintain the fish population at some preferred level, and still allow the exploiters/harvesters to increase their return.

In short, the problem being addressed is a maximization problem of returns subject to the dynamics of the renewable resource. In order to state this optimization problem precisely, the dynamics of the renewable resource and the objective cost function needs to be described.

\subsection{Depensation Model}

A logistic model is a model which describes the evolution of the population size of a species such as bacteria, plants, and animals and is given by

$$
\dot{x}=F(x), \quad x(0)=x_{o}
$$

$x(t)$ is interchangeably called the population size, the stock size, or biomass and denotes the resource which is exploited (or harvested) for economic outcome. $x_{o}$ is the initial population size. Population dynamics are notoriously uncertain and vary drastically from species to species. Furthermore, some population dynamical models may be more appropriate if developed in continuous time for certain species, or in discrete time for others. Some models may need to take into account the spatial distribution of the species, others may need to consider the physical size of the members of the species (such as in fish populations), while others need to include the age structure of the population. Hence, in general $F(x)$ defines a nonlinear partial differential equation. In this article, a depensation model will be considered.

Logistic models are categorized in to three types: Pure compensation models, depensation models, and critical depensation models [35]. In a pure compensation model, $F(x) / x$, called the per capita growth rate, is a non-negative and decreasing function. In a depensation model, $F(x) / x$ is a non-negative function that is increasing for small population sizes and decreasing for large population sizes. In a critical depensation model, $F(x) / x$ is a negative and increasing function for small population sizes, positive and decreasing for large population sizes (see Figure 1). For the most part, pure compensation models have been researched in the literature [36].

In this paper, a depensation model for the population dynamics is considered. The depensation model that will be examined is of the form [36]

$$
\dot{x}=F(x)=r x^{2}\left(1-\frac{x}{k}\right), \quad x(0)=x_{o}
$$


where $r>0$ denotes the intrinsic growth rate and $k>0$ the carrying capacity. Carrying capacity, $k$, represents the maximum population size that the environment can support. Note that when $x=k, F(x)=$ 0 and the population stops growing. For a species, population size may be limited for a variety of reasons such as food availability and competition within the members of the population. In other words, carrying capacity acts as a moderator of the population size.

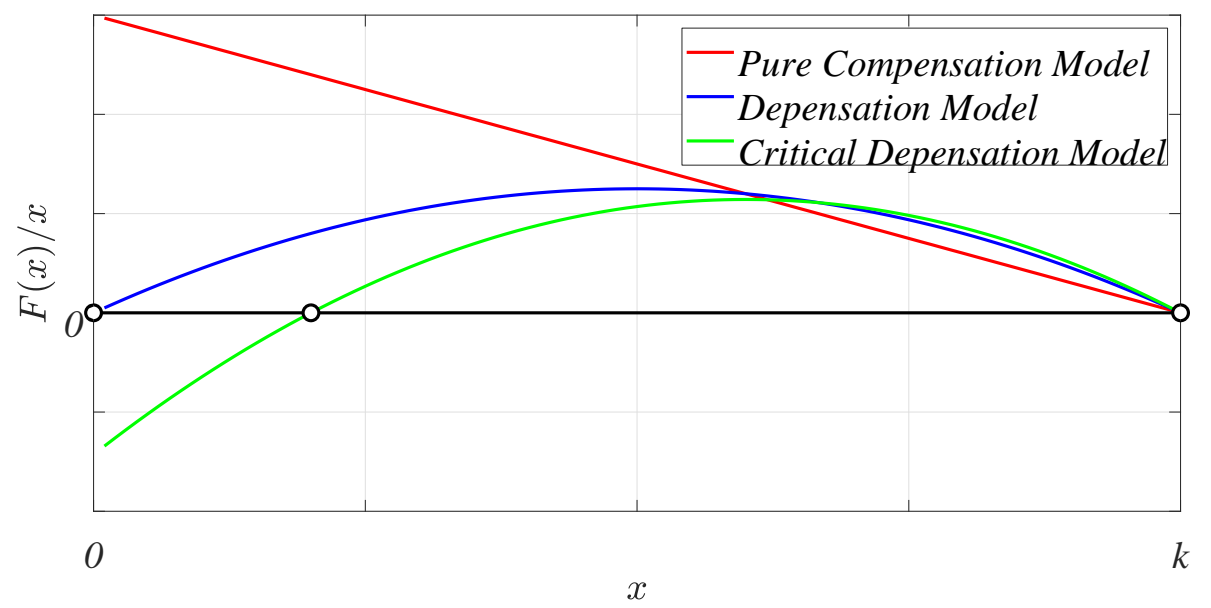

Figure 1. Per capita growth rate functions

Depensation may be due to many reasons, two of which are the species' reproductive behaviour and the spatial distance between the members of the species. Other depensatory models can be found in the literature [37-39]. All of these models, depensatory or not, are utilized to describe the population dynamics of various populations. Depensation models are preferred if, for a given species, small population sizes are slower to recover and the maximum growth rate is skewed to the right [37], i.e. the maximum growth rate is achieved for high population sizes (see Figure 2).

Equation (2) has two natural equilibria at $x=0$ and $x=k$ (see Figure 2). The first equilibrium point at $x=0$ is an unstable equilibrium. For any $x>0$, however small, population size will tend to increase since $F(x)>0$. (Note: $x \leq 0$ is a trivial case, which means there is no resource to exploit. So, for the rest of the paper, the analysis will be limited to $x>0$.) The equilibrium point at $x=k$, on the other hand, is a stable equilibrium. If $x<k$, then $F(x)>0$ and the population size will increase. If for any reason $x>k$, then $F(x)<0$ (not shown in Figure 2) and the population size is driven back towards $k$.

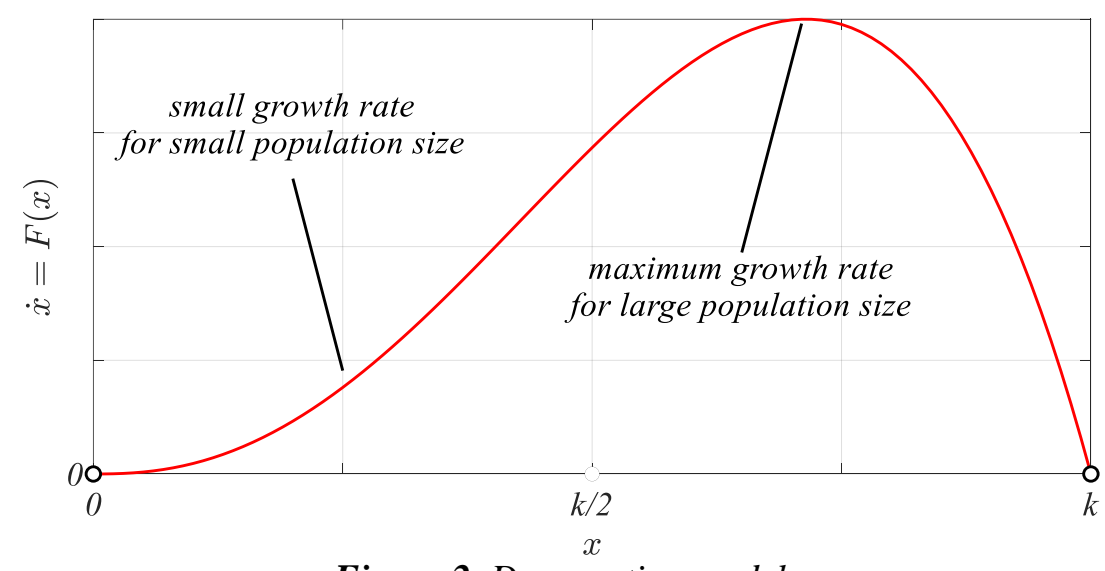

Figure 2. Depensation model

\subsection{Depensation Model with Harvesting}

Let the renewable resource described by Equation (2) is harvested at a rate proportional to the effort spent, such that the modified dynamics takes the form 


$$
\begin{aligned}
\dot{x} & =F(x)-q u x \\
& =r x^{2}\left(1-\frac{x}{k}\right)-q u x, \quad x(0)=x_{o}
\end{aligned}
$$

where $u \in\left[0, u_{\max }\right]$ denotes the effort, or the control action $\left(u_{\max }>0\right)$, and $q>0$ is defined as the catchability coefficient. Figure 3 shows the growth rate function, $F(x)$, and various harvesting curves, $q u x$, separately.

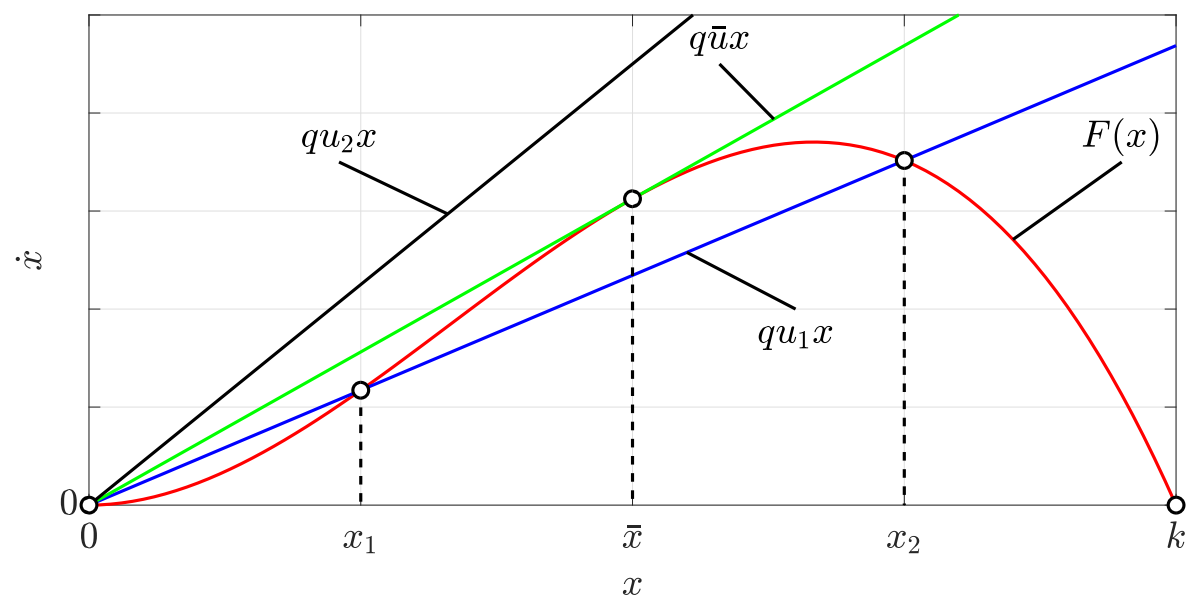

Figure 3. Depensation model subject to harvesting

The roots of Equation (3) are given by

$$
x_{1,2}=\frac{k}{2} \mp \frac{k}{2} \sqrt{1-\frac{4 q u}{k r}} \quad \text { and } \quad x_{3}=0 \text {. }
$$

The magnitude of constant effort, $u$, determines the number of equilibria the harvested depensation model will possess. Regardless of the magnitude of $u$, there is a trivial equilibrium point at $x=0$. For the remaining equilibrium points, there are 3 possible scenarios:

- For a specific magnitude of

$$
\bar{u}=\frac{k r}{4 q}
$$

there is an equilibrium point of multiplicity 2 at $\bar{x}=k / 2$. As shown in Figure 3, this equilibrium is unstable because $F(x)-q \bar{u} x<0$ for all $x$, other than $\bar{x}$. So, a constant effort of $\bar{u}$ will decrease the population size to zero when $x$ is slightly perturbed.

- When $u=u_{2}>\bar{u}$, there are no other real equilibrium points and $F(x)-q u_{2} x<0$ for all $x$, which will reduce the population size to zero.

- When $u=u_{1}<\bar{u}$, there are two distinct equilibrium points. $x=x_{2}$ is a stable equilibrium. Any deviation from $x_{2}$ will drive to population size back to $x=x_{2}$. Note that $F(x)-q u_{1} x<0$ when $x>x_{2}$ and $F(x)-q u_{1} x>0$ when $x=x_{2}$. The other equilibrium at $x=x_{1}$, on the other hand, is unstable. When $x<x_{1}$, the population is driven to extinction. When $x>x_{1}$, the population size increases towards $x=x_{2}$.

The renewable resource manager, i.e., the control system will have to manipulate the effort to maintain the population size at a certain stable level (sustainability), while maximizing some cost function. 


\subsection{Cost Function}

Resources are harvested for economic gain. The question is, how a resource should be exploited to maximize the gain. Let the market price of the resource is denoted by $p$, and the opportunity cost associated with harvesting is denoted by $c$. Then, the net revenue of harvesting (per year) is given by [24]

$$
R=p q u x-c u=(p q x-c) u .
$$

The resource exploiters will manage the resource so as to maximize the total discounted present value of the resource, given by

$$
J \triangleq \int_{0}^{\infty} e^{-\delta t}(p q x-c) u d t
$$

subject to $0 \leq u(t) \leq u_{\max }$ where $\delta$ is the discount rate (annual inflation). So, the optimization problem that needs to solved can now be stated as

$$
\begin{gathered}
\text { maximize } \int_{0}^{T} e^{-\delta t}(p q x-c) u d t \\
\text { such that } \dot{x}=r x^{2}\left(1-\frac{x}{k}\right)-q u x, \quad x(0)=x_{o} \\
0 \leq u(t) \leq u_{\max }
\end{gathered}
$$

\section{MANAGEMENT STRATEGIES/CONTROL LAWS}

Two different control laws to manage the renewable resource will be discussed in this section: An optimal controller based on Pontryagin's Maximum Principle and a sub-optimal model based dynamic linear controller.

\subsection{Optimal Control Law}

The solution to the optimization problem given in Equation (7) is obtained below [40]. Define the Hamiltonian as

$$
\begin{aligned}
\mathcal{H}(x, u, \lambda) & \triangleq e^{-\delta t}(p q x-c) u+\lambda(F(x)-q x u) \\
& =G(x, t) u-\lambda F(x)
\end{aligned}
$$

where

$$
G(x, t) \triangleq e^{-\delta t}(p q x-c)-\lambda q x
$$

and

$$
F(x)=r x^{2}\left(1-\frac{x}{k}\right)
$$

and $\lambda$ represents the co-state variable. Pontryagin's Maximum Principle [41] then yields the following conditions for the optimal control law 


$$
u(t)= \begin{cases}0 & \text { when } \quad G(x, t)<0 \\ u_{\max } & \text { when } \quad G(x, t)>0\end{cases}
$$

Analyzing Equation (12), it can be seen that a singular control arises when the coefficient of $u$ is zero, i.e., when $G(x, t)=0$. Equating

$$
\frac{d \lambda}{d t}=-\frac{\partial \mathcal{H}}{\partial x}
$$

and substituting $G(x, t)=0$ yields [36]

$$
\frac{\partial F(x)}{\partial x}+\frac{c F(x)}{x(p q x-c)}=\delta
$$

of which the numerator can be expanded as

$$
p(x)=3 p q r x^{3}-2 r(c+p q k) x^{2}+k(c r+\delta p q) x-c \delta k=0 .
$$

Since all of the parameters in Equation (15) are positive, using Descartes' rule of signs, it can be shown that $p(x)$ has no real negative roots and has at least one real positive root. When solved for this positive real root, Equation (14) defines an optimal population size $x_{o p t}$, which in turn defines an optimal control effort given by

$$
u_{o p t}=\frac{F\left(x_{o p t}\right)}{q x_{o p t}} .
$$

Finally, the optimal control law can be stated as

$$
u(t)= \begin{cases}0 & \text { when } \quad x<x_{o p t} \\ u_{o p t} & \text { when } \quad x=x_{o p t} \\ u_{\text {max }} & \text { when } \quad x>x_{o p t}\end{cases}
$$

\subsection{Sub-optimal Control Law}

There are many methods available in the control literature to design controllers, such as pole placement, frequency domain loop shaping, model based (state feedback + state observer) compensators, LQR, LQG/LTR, $\mathcal{H}_{\infty}$ mixed-sensitivity, $\mathcal{H}_{2}$, model reference adaptive control, sliding mode, etc. These methods are accompanied by various structures such as P, PI, PID, lead-lag, and more general structures like statespace and transfer functions. Some of these methods are directly applicable to controlling nonlinear systems such as the system described in this paper. One common method of designing a control system for a nonlinear system is to linearize the system at an appropriate equilibrium point and design a linear controller for the linearized system. In this paper, the optimal control strategy will be contrasted with a model based dynamic controller. The aim is to demonstrate how a sub-optimal dynamic controller performs in terms of robustness with respect to parameter uncertainties, disturbances and measurement noise.

First, the model in Equation (3) is linearized about the optimal state and control, $\left(x_{\text {opt }}, u_{\text {opt }}\right)$. The rationale behind the selected linearization point stems from $i$ ) the requirement that the sub-optimal controller is 
expected to perform such that the cost can be made closer to the optimal cost, and ii) the controller is expected to drive the system to $\left(x_{o p t}, u_{o p t}\right)$, and maintain at those values. Linearization yields a first order plant given by

$$
\dot{x}=a x+b u
$$

where

$$
a=\left.\frac{\partial F(x)}{\partial x}\right|_{x=x_{o p t}}-q u_{o p t}=\frac{r x_{o p t}\left(2 k-3 x_{o p t}\right)}{k}-q u_{o p t}, \quad \text { and } \quad b=-q x_{o p t}
$$

Based on the linearized model, a PI controller is designed. Let $k_{p}$ and $k_{i}$ denote the proportional and integral gains of the controller and $e \triangleq x_{o p t}-x$ denote the tracking error. Since the output of the controller needs to be bounded by $0 \leq u(t) \leq u_{\max }$, a clamping integrator anti-wind up feature is included such that $0 \leq k_{p} e+k_{i} \int e \leq u_{\max }$.

The selection of $\left(x_{o p t}, u_{o p t}\right)$ as the linearization point needs to be further discussed because, as it is well known, the linearization holds only in the vicinity of the equilibrium points. Note that depending on $x_{\text {opt }}$ and $u_{\text {opt }}$, the linearized system maybe stable or unstable, i.e. $a$ can be positive or negative. However, $b$ is always negative. Hence, the linearized system is a first order system with a negative gain. For such a system, a stabilizing non-minimum phase PI controller can be designed. Since $b<0, k_{p}$ and $k_{i}$ will be negative.

If $x(t)$ is much smaller than $x_{\text {opt }}$, the error will be positive. Furthermore, the integral of the error will also be positive. Because $k_{p}$ and $k_{i}$ are both negative and $0 \leq u \leq u_{\max }$, the output of the PI controller will be $u=0$. Since $F(x) \geq 0$ for $0 \leq x(t) \leq k$, this will cause $x(t)$ to approach $x_{o p t}$, i.e. $x(t)$ will evolve to fall in the vicinity of $x_{\text {opt }}$. Similarly, if $x(t)$ is much larger than $x_{\text {opt }}$, the error and its integral will both be negative. In this case, the output of the controller will be positive such that $u_{o p t} \leq u \leq u_{\text {max }}$, and drive the system toward $x_{o p t}$ and $u_{o p t}$, hence causing it to fall in the vicinity of $x_{o p t}$ and $u_{o p t}$ (see Figures 12 and 13 in Section 4.2.). As such, the control strategy manages to drive the system towards the linearization point where the linear system is valid.

Controller parameters are determined such that the closed loop system exhibits a small overshoot (i.e. a damping factor larger than 0.7) and a specified $3 \mathrm{~dB}$ closed loop bandwidth is satisfied. In a renewable resource management setting, the controllers cannot be implemented in continuous time, and hence the controller is discretized via Tustin's method.

\section{NUMERICAL RESULTS AND DISCUSSION}

In the analysis below, variables in Table 1 are used.

Table 1. Variables

\begin{tabular}{|c|l|l|}
\hline Symbol & Description & Unit \\
\hline$t$ & Time & year \\
\hline$x(t)$ & Fish biomass & KT \\
\hline$u(t)$ & Harvesting effort & year/year \\
\hline
\end{tabular}

Table 2 provides a list of the parameters along with their nominal values. 
Table 2. Parameters and nominal values

\begin{tabular}{|l|l|l|l|}
\hline Symbol & Description & Unit & Nominal \\
\hline$x_{o}$ & Initial population & $K T$ & - \\
\hline$r$ & Intrinsic growth rate & $1 /$ year & 0.0025 \\
\hline$k$ & Carrying capacity & KT & 200 \\
\hline$q$ & Catchability & $1 /$ year & 0.3 \\
\hline$p$ & Resource market price & $M T L / K T$ & 5 \\
\hline$c$ & Cost of harvesting per & $M T L /$ year & 20 \\
\hline$\delta$ & Annual discount rate & - & 0.1 \\
\hline$T$ & Planning horizon & year & 50 \\
\hline$u_{\min }$ & Minimum effort & year/year & 0 \\
\hline$u_{\max }$ & Maximum effort & year/year & 1 \\
\hline
\end{tabular}

At this point it should be stated that nominal values for these parameters are uncertain with certain bounds, but also subject to change in time. Effect of uncertainties for certain parameters are analyzed in this paper, however, the analysis assumes that the nominal values are not changing in time. This assumption is made, because what is aimed in this study is to compare the properties of the optimal and sub-optimal strategies. Of course, in a realistic setting, strategies are not expected to remain valid for 50 years, and nominal values are estimated periodically and taken into consideration in updating the control design.

\subsection{Optimal Control Law}

For the parameters given in Table 2, the optimal population size and the optimal effort for the optimal control law are computed to be

$$
x_{\text {opt }} \approx 113.702 \mathrm{KT} \text { and } u_{\text {opt }} \approx 0.409 \frac{\text { year }}{\text { year }} .
$$

The value of $u_{\text {opt }}$ should be interpreted as the ratio of time spent fishing per year. So, $u_{\text {opt }} \approx 0.409$ roughly corresponds to 5 months of fishing per year.

Simulations were run for $x_{o}=50 \mathrm{KT}$ and $x_{o}=150 \mathrm{KT}$. Trajectories for $u(t)$ and $x(t)$ are given in Figures 4 and 5, respectively.

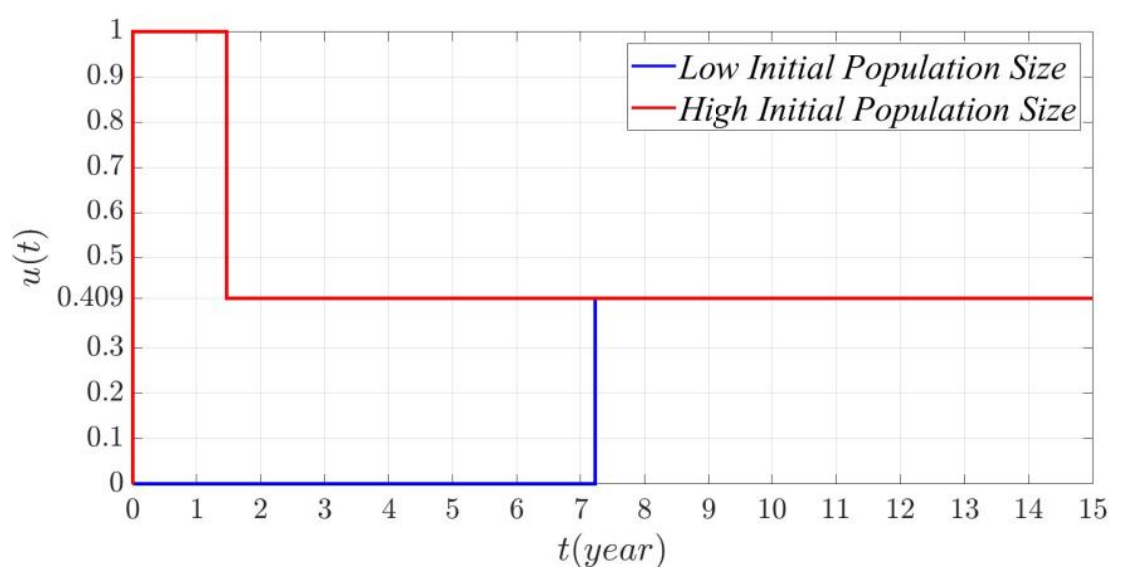

Figure 4. Optimal control: fishing effort $u(t)$ yearlyear

As expected from the optimal control law, when the population size is larger than the computed optimal value, fishing effort is set to its maximum. This causes the population to decrease from its initial value. 
Once the optimal population level is reached, fishing effort is set to $u_{\text {opt }}$, and the population size is maintained at $x_{\text {opt }}$.

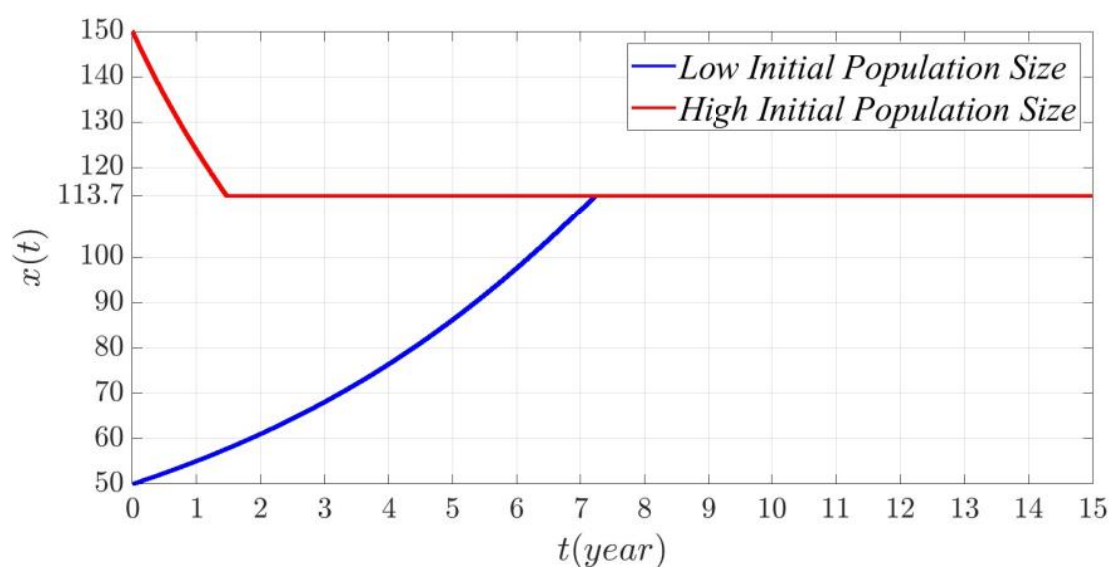

Figure 5. Optimal control: population size $x(t) K T$

As for the small initial population case, since $x<x_{\text {opt }}$ fishing effort is set to zero. This causes the population to recover and increase from its initial value. Once the optimal population level is reached, fishing effort is set to $u_{o p t}$, and the population size stays constant at $x_{o p t}$.

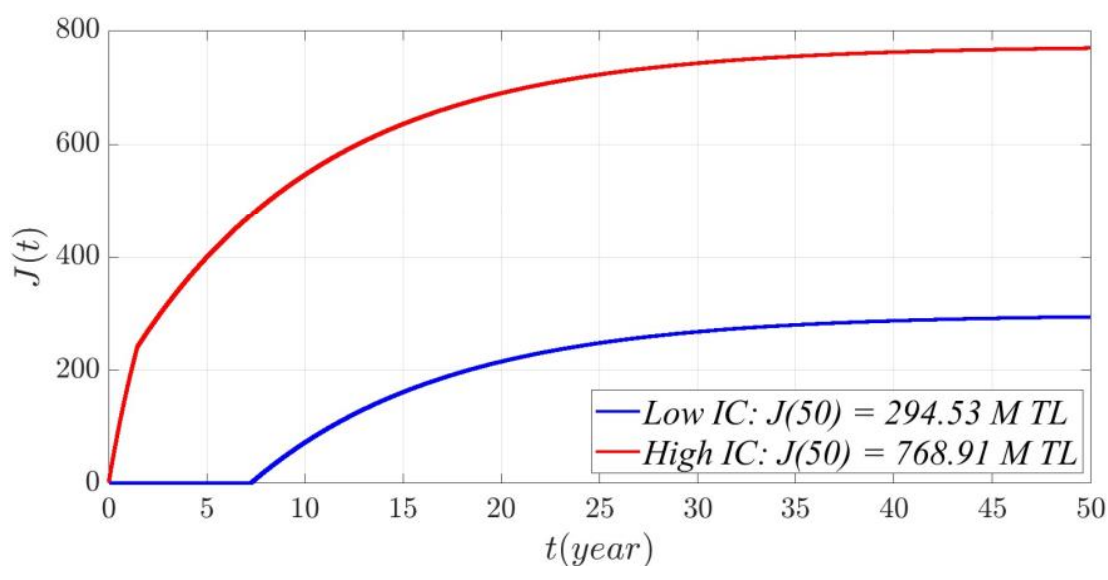

Figure 6. Optimal control: discounted present value $J(t) M T L$

Figure 6 shows the discounted present value, i.e., the objective function vs. time after 50 years when all parameters are assumed fixed. Total revenue is $\approx 294.5 \mathrm{M}$ TL for the low initial population scenario, and $\approx$ 768.9 M TL for the high initial population case. The main reason why the low initial condition case is drastically smaller is the discount rate, i.e. inflation. It takes about 7 years for the fishing activity to start, by which time discounted return drops significantly.

When the parameters are assumed to be certain and when there are no disturbances or measurement noise, optimal control law performs as expected and returns the optimal revenue, as shown in Figures 4, 5 and 6.

Implementation of controllers: A typical standard negative feedback system structure is given in Figure 7. 


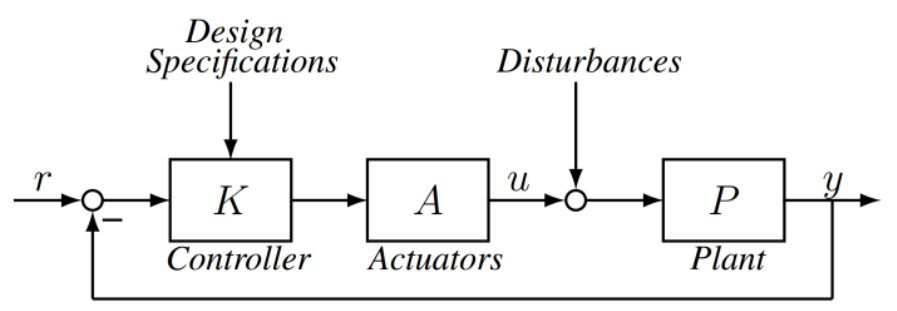

Figure 7. Feedback Diagram

In designing control systems, it is accepted that the plant to be controlled is uncertain, some of its dynamics are neglected, and disturbances are unknown. However, control engineers are typically certain about the actuator and controller dynamics. These controllers are implemented either on a circuit board, or as it is mostly the case, are programmed on micro-controllers or FPGAs.

Renewable resource management, on the other hand, is usually conducted by governments and/or institutions and they lack the possibility to implement strategies in this fashion. Institutions themselves have uncertain dynamics. This is why a big fraction of the renewable source management research is dedicated to modeling and understanding institutions and the uncertainties associated with them [42-45]. In Figure 8, the same feedback structure is given with the blocks' new meaning in the resource management context.

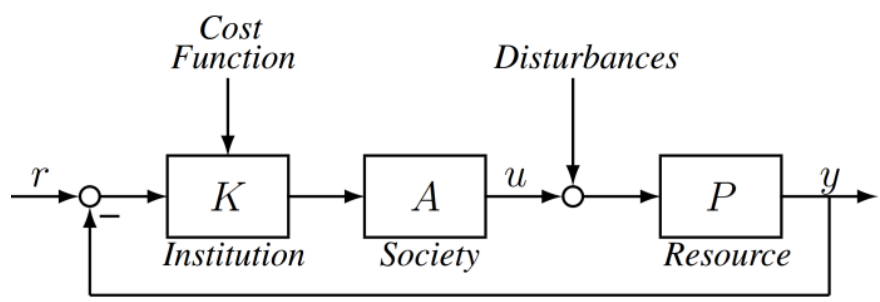

Figure 8. Feedback Diagram - Institutional Context

For an institution, implementing the optimal controller, or as it is phrased in sustainability community, the optimal policy requires certain tools. It is easy to ban fishermen from fishing. However, the institution still needs to monitor and make sure that the ban is enforced. If an institution wants less fishing effort, it cannot enforce the policy directly on fishermen. It either implements quotas, or increases taxes to increase the opportunity cost. Yet, implementation may still not be as desired. In short, for a renewable resource management problem, not only the plant but also the controller and actuators are uncertain.

The analysis below is aimed at demonstrating the implementation issues associated with the optimal control of a renewable resource. In the subsequent section, the same analysis will be performed on the sub-optimal dynamic controller. Referring to Figures 7 and 8, the management strategies (controllers) are represented by the Institution/Controller block, population dynamics are represented by the Resource/Plant block. Societal dynamics/actuators are outside the scope of this study, and therefore they will be assumed identity. Examples will include parametric uncertainties, measurement noise, and disturbances.

In the analyses below, the optimal controller is designed (i.e. $x_{\text {opt }}$ and $u_{\text {opt }}$ are determined) based on the nominal values given in Table 2 and Equation (17) in which $x_{o p t}$ and $u_{o p t}$ are computed by Equations (15) and (16). The performance of the nominal optimal controller is evaluated. In order to assess the performance of the optimal controller, parametric uncertainty is treated as plant uncertainty. In other words, the controller, designed for the nominal system, is acting on a perturbed system. A similar strategy is applied for disturbances and measurement noise.

Parametric uncertainties: All of the parameters in the depensation model of the population, harvesting model and the cost function are uncertain and subject to change. Cost $(c)$, market price $(p)$, and discount 
rate $(\delta)$ can possibly be estimated with some level of certainty, but they are by no means certain. The biological parameters on the other hand are notoriously difficult to estimate, simply because estimating those parameters in a very large medium (such as the open seas) is too expensive and the interactions of a species with its ecology is too complex to be modeled with certainty.

In order to demonstrate the vulnerability of the optimal controller to parametric uncertainties, a $1 \%$ estimation error is introduced for the carrying capacity parameter. Note that, in a realistic setting, $1 \%$ estimation error for carrying capacity parameter is very optimistic. Typically, the uncertainty associated with the carrying capacity would be much higher. Figure 9 shows that even a small uncertainty in a parameter causes the optimal fishing effort (control action) to go into a limit cycle like behavior. This type of fishing effort is virtually impossible to enforce or implement. As shown in Figure 9, implementing the optimal controller requires a large number of switching action between $u=0, u=u_{\text {opt }}$, and $u=1$. Considering that the control effort corresponds to the amount of time spent for harvesting the resource by fishermen, changing the rules for fishing more than 30 times a year is an impossible feat. This is equivalent to expecting good command following performance from a system at a frequency higher than its bandwidth.

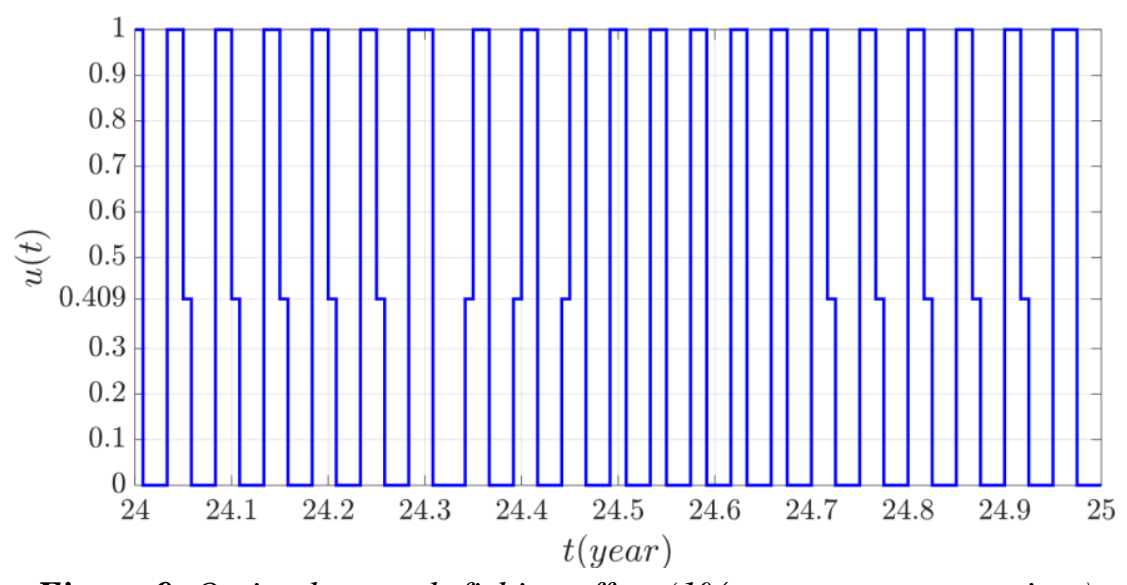

Figure 9. Optimal control: fishing effort (1\% parameter uncertainty)

Measurement errors: Measurement errors are inevitable. Populations of wild species, such as fish, are measured through a variety of ways [46]. Some include sonars and aerial monitoring. However, the most common method is through the amount of fish caught. Estimation of the total population size via the harvested amount assumes that a known fraction of the total population can be harvested. The parameter that determines this fraction is the catchability rate, $q$.

Figure 10 focuses on a single year of the control (harvesting) effort response of the optimal controller subject to noisy measurement. Introduced noise has mean zero and variance 0.04 . Given the range of $x \in$ $(0,200]$, this measurement noise is very small. As shown, even a very small magnitude of noise renders the fishing effort impossible to implement for the reasons explained in the parametric uncertainty scenario above, i.e. the switching action required between $u=0, u=u_{o p t}$, and $u=1$ at a high frequency. 


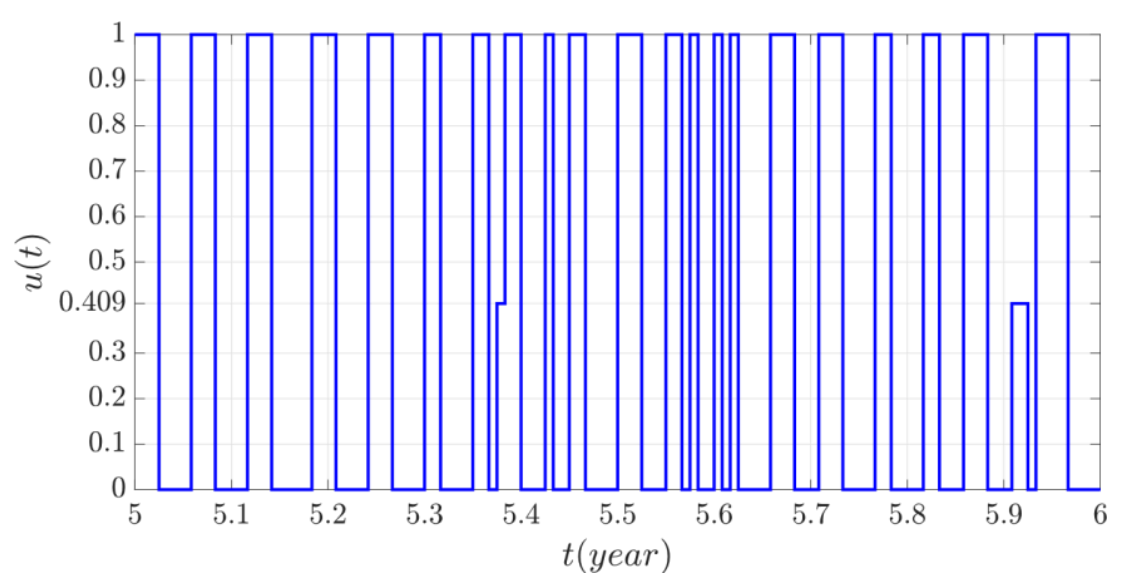

Figure 10. Optimal control: fishing effort (noisy measurement)

Input disturbances: Disturbances at the plant input effect the controller output directly. In the renewable resource management setting, one source of input disturbances corresponds to the fishermen not abiding by the rules. In the simulations below, it was assumed that even though the fishermen are urged to fish at $u=u_{\text {opt }} \approx 0.409$, they are fishing $1 \%$ more, i.e. either cheating only $1 \%$ or making an honest mistake of fishing longer than the allowed period. As shown in Figure 11, the effort response displays a limit cycle like behavior. For similar reasons explained in the parametric uncertainty and measurement noise scenarios, this type of high frequency control effort is almost impossible to implement on the field.

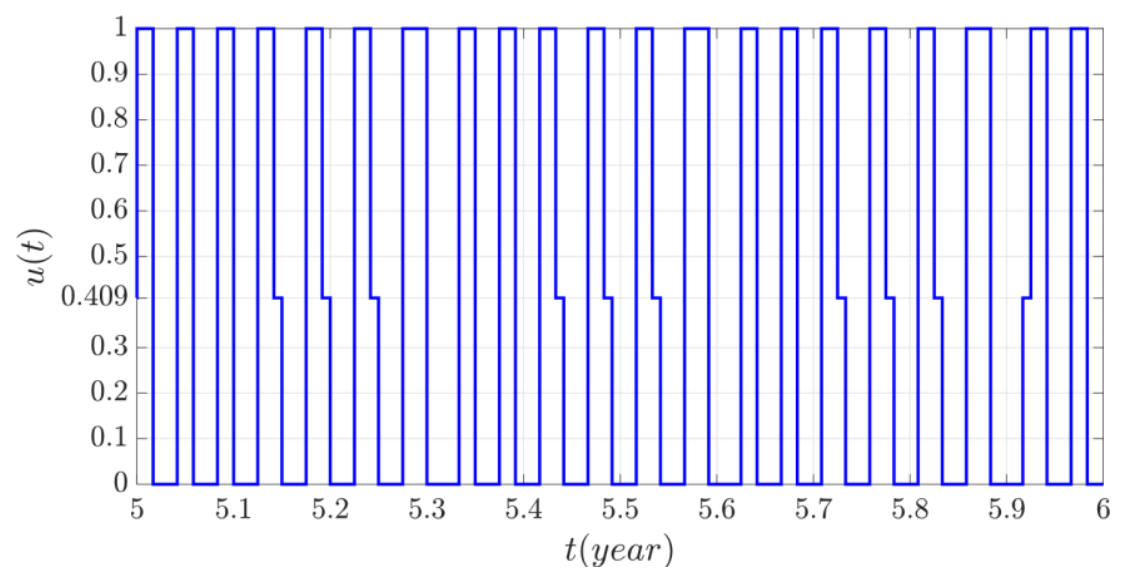

Figure 11. Optimal control: fishing effort (1\% cheating)

The above analysis tries to demonstrate the vulnerabilities of the optimal controller. It was aimed to show that, very small uncertainties in the parameters, very small measurement noise, or a very small input disturbance cause the optimal control strategy to fail due to implementation issues. The main reason behind this vulnerability is the infinite gain nature of the optimal control law. From a control systems perspective, the optimal controller is a bang-bang controller and the problems associated with it are well known. Optimal controller responds to any deviation from the optimal population size, however small, aggressively and causes the system to oscillate at a high frequency.

What is really required is a controller (strategy) that is easy to implement, that is not sensitive to small deviations, that converges to the optimal population size in a smooth and implementable fashion, and also provides predictability for the future so that harvesting the resource can be considered secure source of income. 


\subsection{Model Based Dynamic Control Law}

The structure and methodology of the model based control law designed for the renewable resource dynamics presented in this paper was explained in Section 3.2. The resulting controller includes proportional and integral components, but it is nonlinear in the sense that the output is saturated and an integrator anti-wind up procedure is included.

For the nominal case where the parameters are certain, measurement noise and the disturbances are zero, and in continuous time, population $(x(t))$ and effort $(u(t))$ graphs are given in Figures 12 and 13 . As expected form a dynamic finite gain controller, there is no switching action in the control effort. The control system allows for overshoot and gradually achieves command following.

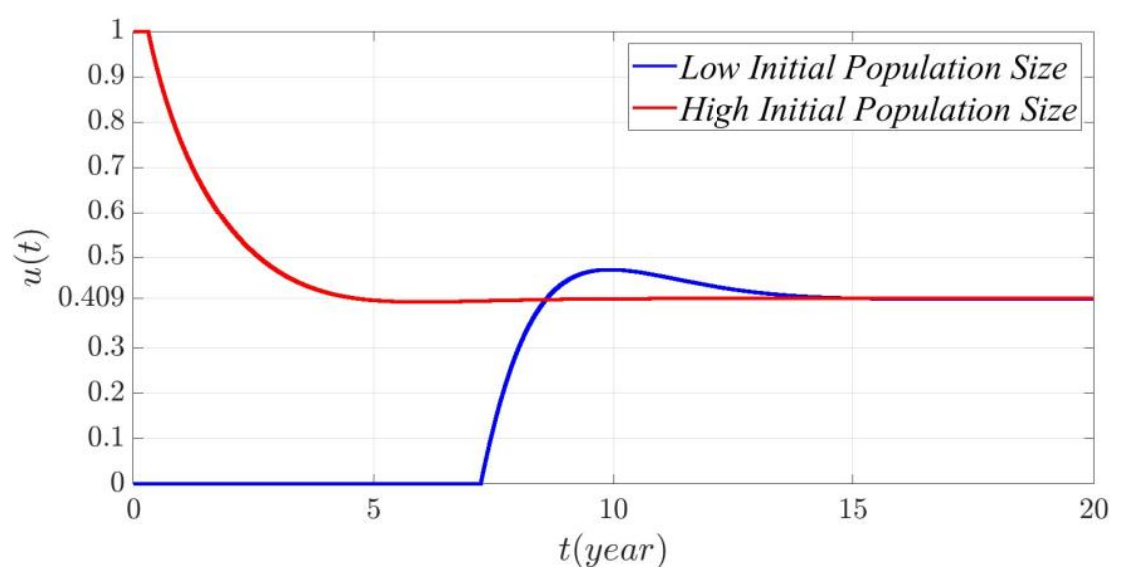

Figure 12. Dynamic control: fishing effort $u(t)$ year/year

After 50 years, the total revenue is $\approx 294.1 \mathrm{M}$ TL for the low initial population case, and $\approx 768.2 \mathrm{M}$ TL for the high initial population case. As expected, the cost function is below the theoretical optimum, although slightly. ( $\approx 294.5 \mathrm{M}$ TL for the low initial population case, and $\approx 768.9 \mathrm{M}$ TL for the high initial population case.)

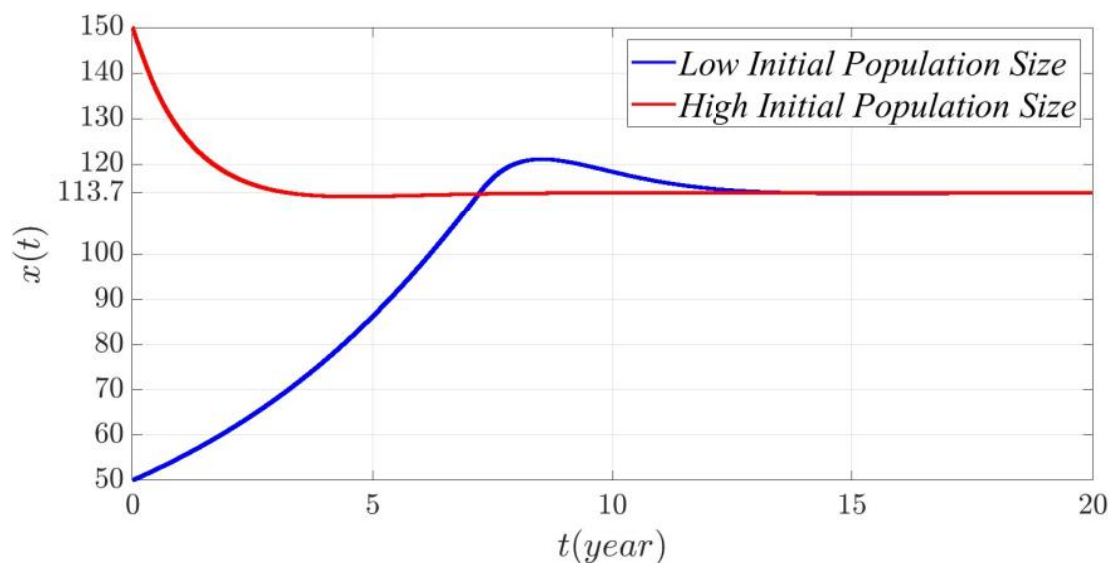

Figure 13. Dynamic control: population size

It is, then, demonstrated that a dynamic control law can be designed such that the economic objectives (cost function) of the original optimal control problem may be achieved within a small margin. Similar to the optimal controller analysis, dynamic controller will be analyzed subject to parametric uncertainties, measurement noise, and disturbances. In the analyses below, the PI controller is designed (i.e. $x_{o p t}, u_{o p t}, k_{p}$ and $k_{i}$ are determined) based on the nominal values given in Table 2 and Equation (17) in which $x_{\text {opt }}$ and $u_{\text {opt }}$ are computed Equations (15) and (16). The performance of the nominal PI controller is evaluated. As 
in the case of the optimal controller assessment case, in order to assess the performance of the PI controller, parametric uncertainty is treated as plant uncertainty. In other words, the controller, designed for the nominal system, is acting on a perturbed system. A similar strategy is applied for disturbances and measurement noise.

Parametric uncertainties: In Figure 9, it was demonstrated that a 1\% estimation error in $k$, the carrying capacity parameter, optimal controller performed poorly in terms of the control effort that needs to be implemented. Figure 14 demonstrates that to a $\pm 20 \%$ estimation error in $k$ the dynamic controller is very robust in the sense that the control effort is apt to implementation in a renewable resource management setting and as shown in Figure 15, the population size converges to $x_{\text {opt }}$. Furthermore, it performs better than the optimal controller in terms of the cost if the parameter is under estimated. This should not be surprising because, note that when parameters are varied theoretical optimality is lost.

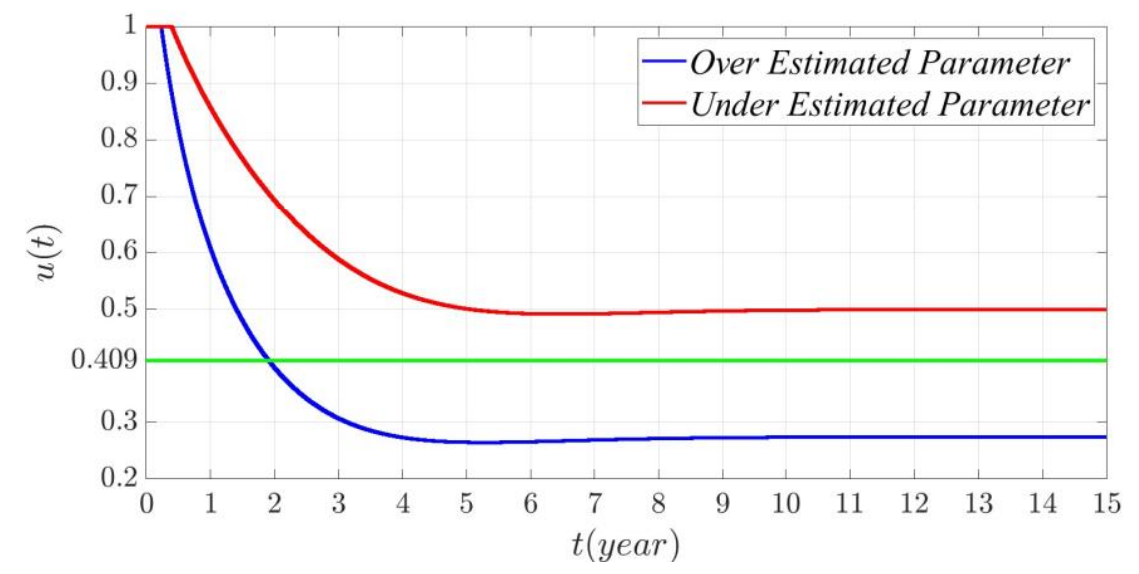

Figure 14. Dynamic control: fishing effort ( $\pm 20 \%$ parameter uncertainty)

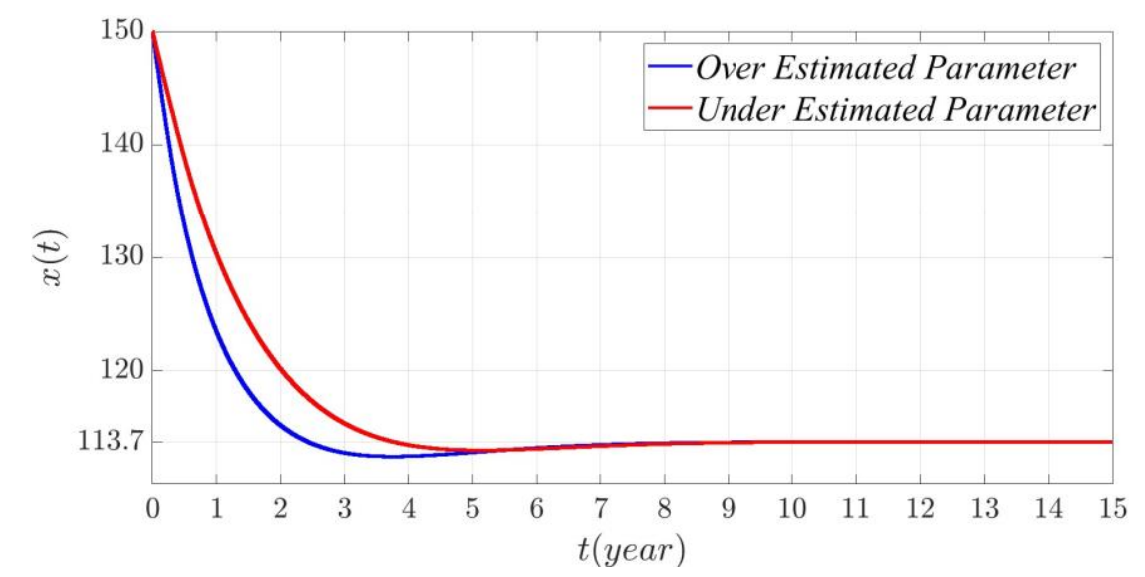

Figure 15. Dynamic control: population size ( $\pm 20 \%$ parameter uncertainty)

Measurement errors: Subject to the same measurement errors as the optimal controller, dynamic controller's output does not oscillate wildly between $u_{\text {min }}, u_{\text {opt }}$, and $u_{\max }$ as shown in Figure 10 . The impact of error is still visible in the control (fishing) effort time response. However, the effect of measurement noise does not cause the effort to oscillate but instead, introduces noise in the control effort (Figure 16). The shown control effort may not be practically implemented as is, however, it may still be implemented after some smoothing operation, such as a moving average. 


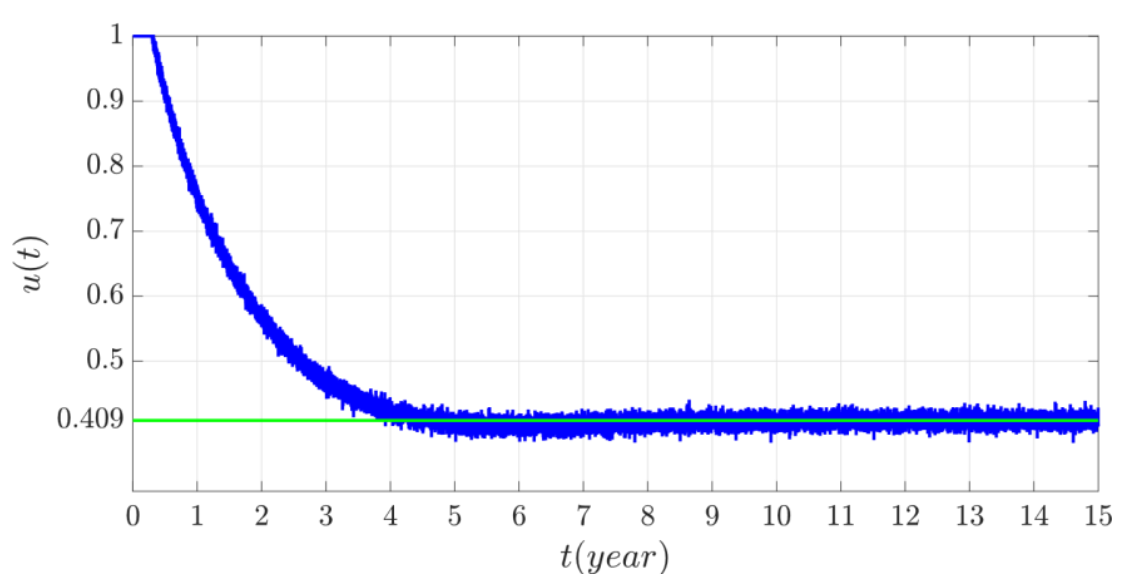

Figure 16. Dynamic control: fishing effort (noisy measurement)

Input disturbances: In Figure 11, optimal control was shown to be problematic when subject to constant input disturbances, when a disturbance analogous to a $1 \%$ cheating or honest mistake by the fishermen was applied. Dynamic controller is now tested when there is $20 \%$ cheating/mistake. Figure 17 shows that dynamic controller handles the disturbance by appropriately reducing the control effort demand (Note that the steady state control effort is around 0.33 , which as roughly $20 \%$ smaller than the optimal value.).

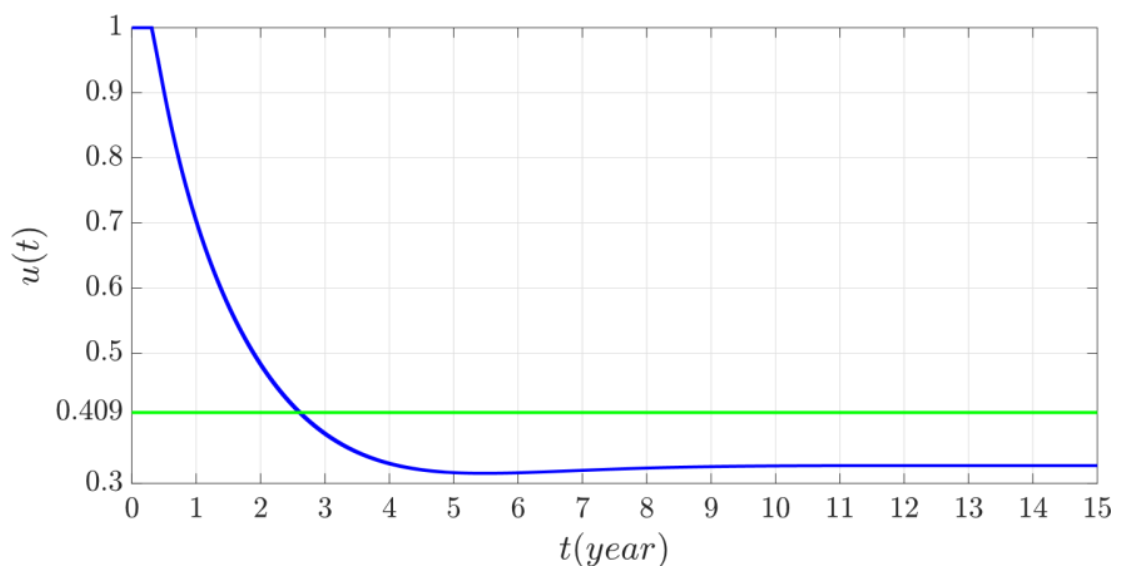

Figure 17. Dynamic control: fishing effort (20\% cheating)

The above analysis demonstrates that the tools and methods routinely utilized by control engineers in the field of electrical engineering can readily be used for social-ecological. A dynamic controller proves much more robust to the disturbances, uncertainties and measurement noise that are encountered in renewable resource management problems. Robustness is to be understood in the sense that the population size of the resource is maintained at the desired population level, and the control effort that needs to be applied in order to achieve the desired population size is practically implementable even when subject to measurement noise, parametric uncertainties, and disturbances. Dynamic controller, although costlier than the optimal controller in terms of computing, essentially requires the integration of the error between the measured population size and the desired population size. Saturating the commanded control effort and clamping the integrator to prevent wind-up issues can be implemented fairly easily. In other words, a dynamic-controllertype data processing would give the resource managers much more reliable data and guidance for the resource to be managed in a sustainable way.

\section{CONCLUSION AND FUTURE DIRECTIONS}

Many fisheries have collapsed around the world and the management strategies employed are to blame. The main reason for these strategies to fail is due to uncertainties in resource models and practical limitations of implementing policies. In this paper, a depensation type resource model, along with a bio- 
economic cost function is considered. From the perspective of control systems design, an optimal control law is formulated. The deficits of the optimal control law are demonstrated and a sub-optimal control law is proposed. It was shown that a sub-optimal controller performed much better when subject to model uncertainties, measurement noise, and input disturbances. It is argued that the tools and methods of robust control systems could provide a guidance into renewable resource management problems. It was stated that the main difference between a control system designed to control a physical system and an ecological system is the uncertainty associated with the implementation of the control designs. In a social-ecological setting, even though the control algorithm is predetermined, implementation involves uncertain controllers (institutions) and uncertain actuators (societies).

Depensation type resource models are one but a variety of models that describe renewable resources. Every species must be modeled according to their spatial distributions, age structures, mating habits, growth cycles, interactions with other species, and also their interactions with the environment. The better the model, the better the performance of control. Future work should consider different types of resource models, better parameter estimation and measurement methods, and more complex and robust control laws.

\section{CONFLICTS OF INTEREST}

No conflict of interest was declared by the author.

\section{REFERENCES}

[1] Clark, C.W., "Fisheries bioeconomics: why is it so widely misunderstood?", Population Ecology, 48(2): 95-98, (2006).

[2] Clark, C.W., Munro, G.R., Sumaila, U.R., "Subsidies, buybacks, and sustainable fisheries", Journal of Environmental Economics and Management, 50(1): 47-58, (2005).

[3] Holland, D., Gudmundsson, E., Gates, J., "Do fishing vessel buyback programs work: a survey of the evidence", Marine Policy, 23(1): 47-69, (1999).

[4] Myers, R.A., Worm, B., "Rapid worldwide depletion of predatory fish communities", Nature, 423(6937): 280-283, (2003).

[5] Sethi, G., Costello, C., Fisher, A., Hanemann, M., Karp, L., "Fishery management under multiple uncertainty", Journal of Environmental Economics and Management, 50(2): 300-318, (2005).

[6] Moran, E.F., Ostrom, E., "Seeing the forest and the trees: human-environment interactions in forest ecosystems", The MIT Press, Massachusetts, (2005).

[7] Fuchigami, Y., Hara, K., Uwasu, M., Kurimoto, S., "Analysis of the mechanism hindering sustainable forestry operations: a case study of Japanese forest management", Forests, 7(8): 182, (2016).

[8] Cunniffe, N.J., Cobb, R.C., Meentemeyer, R.K., Rizzo, D.M., Gilligan, C.A., "Modeling when, where, and how to manage a forest epidemic, motivated by sudden oak death in California", Proceedings of the National Academy of Sciences, 113(20): 5640-5645, (2016).

[9] Pauloo, R.A., Escriva-Bou, A., Dahlke, H., Fencl, A., Guillon, H., Fogg, G.E., "Domestic well vulnerability to drought duration and unsustainable groundwater management in California's central valley", Environmental Research Letters, 15(4): 044010, (2020). 
[10] Rinaudo, J.D., Donoso, G., "State, market or community failure? Untangling the determinants of groundwater depletion in Copiapo (Chile)", International Journal of Water Resources, 35(2): 283304, (2019).

[11] Gorelick, S.M., Zheng, C., "Global change and the groundwater management challenge", Water Resources Research, 51(5): 3031-3051, (2015).

[12] Powlson, D.S., Gregory, P.J., Whalley, W.R., Quinton, J.N., Hopkins, D.W., Whitmore, A.P., Hirsch, P.R., Goulding, K.W.T., "Soil management in relation to sustainable agriculture and ecosystem services", Food Policy, 36: 72-87, (2011).

[13] Juerges, N., Hansjurgens, B., "Soil governance in the transition towards a sustainable bioeconomy - a review", Journal of Cleaner Production, 170: 1628-1639, (2018).

[14] Clark, C.W., Kirkwood, G.P., "On uncertain renewable resource stocks: optimal harvest policies and the value of stock surveys", Journal of Environmental Economics and Management, 13(3): 235-244, (1986).

[15] Koenig, E.F., "Controlling stock externalities in a common property fishery subject to uncertainty", Journal of Environmental Economics and Management, 11(2): 124-138, (1984).

[16] Belkhodja, K., Moussaoui, A., Alaoui, M.A.A., "Optimal harvesting and stability for a preypredator model", Nonlinear Analysis-Real World Applications, 39: 321-336, (2018).

[17] Dubey, B., Agarwal, S., Kumar, A., "Optimal harvesting policy of a prey-predator model with Crowley-Martin-type functional response and stage structure in the predator", Nonlinear AnalysisModelling and Control, 23(4): 493-514, (2018).

[18] Demir, M., "Optimal control strategies in ecosystem-based fishery models", Phd. Thesis, University of Tennessee, USA, 11-44, (2019).

[19] Huang, L.R., Cai, D.H., Liu, W.Y., "Optimal harvesting of an abstract population model with interval biological parameters", Advances in Difference Equations, 2020(1): 285, (2020).

[20] Kazmi, K.R., Dubey, B., Patra, A., "A mathematical model for optimal management and utilization of a renewable resource by population", Journal of Mathematics, 2013: 613706, (2013).

[21] Ludwig, D., Walters, C.J., "Optimal harvesting with imprecise parameter estimates”, Ecological Modelling, 14(3): 273-292, (1982).

[22] Weitzman, M.L., "Landing fees vs harvest quotas with uncertain fish stocks", Journal of Environmental Economics and Management, 43(2): 325-338, (2002).

[23] Andersen, P., "Commercial fisheries under price uncertainty", Journal of Environmental Economics and Management, 9(1): 11-28, (1982).

[24] Clark, C.W., "The worldwide crisis in fisheries: economic models and human behavior", Cambridge University Press, New York, (2006).

[25] Crepin, A.S., Norberg, J., Maler, K.G., "Coupled economic-ecological systems with slow and fast dynamics - modelling and analysis method", Ecological Economics, 70: 1448-1458, (2011).

[26] Pei, Y.Z., Chen, M.M., Liang, X.Y., Li, C.G., "Model-based on fishery management systems with selective harvest policies", Mathematics and Computers in Simulation, 156: 377-395, (2019). 
[27] Anderies, J.M., Rodriguez, A.A., Janssen, M.A., Cifdaloz, O., "Panaceas, uncertainty, and the robust control framework in sustainability science", Proceedings of the National Academy of Sciences, 104(39): 15194-15199, (2007).

[28] Rodriguez, A.A., Cifdaloz, O., Anderies, J.M., Janssen, M.A., Dickeson, J., "Confronting management challenges in highly uncertain natural resource systems: a robustness-vulnerability trade-off approach”, Environmental Modeling \& Assessment, 16(1): 15-36, (2011).

[29] Bisson, A., Casenave, C., Boudsocq, S., Daufresne, T., "Maximization of fertility transfers from rangeland to cropland: the contribution of control theory", Journal of Theoretical Biology, 469: 187-200, (2019).

[30] Das, D., Kar, T.K., "Feedback control and its impact on generalist predator-prey system with prey harvesting", Nonlinear Analysis-Modelling and Control, 24(5): 718-732, (2019).

[31] De Giovanni, D., Lamantia, F., "Dynamic harvesting under imperfect catch control", Journal of Optimization Theory and Applications, 176(1): 252-267, (2018).

[32] Anderies, J.M., Janssen, M.A., "Robustness of social-ecological systems: implications for public policy”, Policy Studies Journal, 41(3): 513-536, (2013).

[33] Anderies, J.M., Folke, C., Walker, B., Ostrom, E., “Aligning key concepts for global change policy: robustness, resilience, and sustainability", Ecology and Society, 18(2): 8, (2013).

[34] Schoon, M.L., Cox, M.E., "Understanding disturbances and responses in social-ecological systems", Society and Natural Resources, 25(2): 141-155, (2012).

[35] Brauer, F., Castillo-Chavez, C., "Mathematical models in population biology and epidemiology", Springer, New York, (2012).

[36] Clark, C.W., "Mathematical Bioeconomics", John Wiley \& Sons, Inc., New York, (1990).

[37] Liermann, M., Hilborn, R., "Depensation: evidence, models and implications", Fish and Fisheries, 2: 33-58, (2001).

[38] Chan, M.H., Kim, P.S., "Modelling the impact of marine reserves on a population with depensatory dynamics", Bulletin of Mathematical Biology, 76: 2122-2143, (2014).

[39] Saha, B., Bhowmick, A.R., Chattopadhyay, J., Bhattacharya, S., "On the evidence of an Allee effect in herring populations and consequences for population survival: A model-based study", Ecological Modelling, 250: 72-80, (2013).

[40] Knowles, G., "An introduction to applied optimal control”, Academic Press, New York, (1981).

[41] Pontryagin, L.S., Boltyanskii, V.G., Gamkrelidze, R.V., Mishchenko, E.F., "The mathematical theory of optimal processes", CRC Press, New York, (1986).

[42] Anderies, J.M., Mathias, J.D., Janssen, M.A., "Knowledge infrastructure and safe operating spaces in social-ecological systems", Proceedings of the National Academy of Sciences, 116: 5277-5284, (2019).

[43] Harris, M.M., Schaubroeck, J., "Confirmatory modeling in organizational behavior/human resource management: issues and applications", Journal of Management, 16: 337-360, (1990). 
[44] Yang, Z., Su, C., "Understanding Asian business strategy: modeling institution-based legitimacyembedded efficiency", Journal of Business Research, 66(12): 2369-2374, (2013).

[45] Schluter, M., Mcallister, R.R.J., Arlinghaus, R., Bunnefeld, N., Eisenack, K., Hölker, F., MilnerGulland, E.J., Müller, B., Nicholson, E., Quaas, M., Stoven, M., "New horizons for managing the environment: a review of coupled social-ecological" systems modeling", Natural Resource Modeling, 25: 219-272, (2012).

[46] Smith, T.D., "Scaling fisheries: the science of measuring the effects of fishing", 1855-1955, Cambridge University Press, New York, (1994). 